\title{
Avaliação eletromiográfica da sucção em bebês com síndrome de Down
}

\section{Electromyographic evaluation of sucking in infants with Down syndrome}

\author{
Patricia Noriko Ideriha ${ }^{1}$, Suelly Cecília Olivan Limongi ${ }^{2}$
}

\begin{abstract}
RESUMO
Objetivo: Verificar a eficácia do trabalho fonoaudiológico na função da sucção em bebês com síndrome de Down por meio da eletromiografia de superfície. Métodos: Em cada grupo (pesquisa e controle) participaram cinco bebês do gênero masculino e três do feminino, com idades entre seis e dez meses, diferenciando-se pelo diagnóstico da síndrome de Down. A pesquisa foi realizada em três fases: a) avaliação inicial (avaliação clínica do sistema estomatognático e de eletromiografia de superfície - provas de repouso habitual, sucção de mamadeira e retirada de papinha da colher); b) processo terapêutico; c) reavaliação. O grupo controle participou apenas da primeira e terceira fases. Resultados: Os dados eletromiográficos mostraram diferença estatisticamente significante entre as provas de repouso habitual e mamadeira/papinha. No repouso habitual, recrutam-se poucas unidades motoras, em comparação aos demais movimentos realizados pelos músculos. Conclusão: A eletromiografia de superfície não permitiu verificar a diferença da contração do músculo orbicular da boca, antes e após a terapia, nem entre os grupos pesquisados, pois os movimentos não recrutaram unidades motoras suficientes para detectar as diferenças esperadas. Entretanto observou-se melhora dos aspectos observados através da avaliação fonoaudiológica clínica.
\end{abstract}

Descritores: Eletromiografia/utilização; Síndrome de Down; Lactente; Comportamento de sucção

\section{INTRODUÇÃO}

Os exames de eletromiografia (EMG) têm sido usados há mais de 40 anos, proporcionando uma avaliação objetiva e precisa, determinando as características elétricas de um músculo ou de um grupo muscular ${ }^{(1)}$.

Atualmente, a EMG de superfície é utilizada, tanto em aplicações clínicas, quanto em pesquisas, na realização de avaliação neuromuscular não invasiva, em vários campos distintos, como a ciência do esporte, a neurofisiologia e a reabilitação ${ }^{(2)}$. Em complemento, a EMG tem indicação, também, de auxílio na escolha do tratamento a ser indicado, uma vez que contribui com achados objetivos ${ }^{(3)}$.

Muitas pesquisas utilizam o eletromiógrafo, relacionando-o com estruturas orofaciais, com o objetivo de correla-

(1) Mestre, Fonoaudióloga clínica e colaboradora do Laboratório de Investigação Fonoaudiológica em Síndromes e Alterações Sensório-Motoras do Departamento de Fisioterapia, Fonoaudiologia e Terapia Ocupacional da Faculdade de Medicina da Universidade de São Paulo - USP - São Paulo (SP), Brasil.

(2) Livre-Docente, Professora Associada do Departamento de Fisioterapia, Fonoaudiologia e Terapia Ocupacional da Faculdade de Medicina da Universidade de São Paulo - USP - São Paulo (SP), Brasil.

Trabalho realizado no Laboratório de Investigação Fonoaudiológica em Síndromes e Alterações Sensório-Motoras do Departamento de Fisioterapia, Fonoaudiologia e Terapia Ocupacional da Faculdade de Medicina da Universidade de São Paulo - USP - São Paulo (SP), Brasil.

Endereço para correspondência: Patrícia Noriko Ideriha. R. Chile, 275, Jd. das Laranjeiras, Bragança Paulista - SP, CEP 01290-000. E-mail: fga_patricia@hotmail.com

Recebido em: 7/5/2007; Aprovado em: 30/8/2007 cionar as atividades musculares dos lábios superior e inferior durante movimentos orais ${ }^{(4)}$; analisar a atividade muscular do músculo orbicular da boca, em crianças com respiração nasal e oral ${ }^{(5)}$; analisar a atividade muscular labial durante três atividades de deglutição( ${ }^{(6)}$; estudar as diferenças morfológicas em indivíduos com competência e incompetência labial ${ }^{(7)}$; comparar as atividades musculares dos lábios de crianças com e sem fissura labial ${ }^{(8)}$; verificar a eficácia do biofeedback no tratamento de indivíduos adultos com gagueira $^{(9)}$.

Apesar desta variedade de pesquisas, poucas estudam a sucção de bebês, podendo ser citada a análise dos músculos mastigatórios em bebês que utilizam mamadeira ${ }^{(10)}$; análise da atividade dos músculos temporal, masseter, orbicular da boca e supra-hióideo (unilateralmente) durante a alimentação ${ }^{(11)}$ ou dos músculos masseter, temporal e bucinador em situação de aleitamento natural, comparando com o artifici$\mathrm{al}^{(12)}$; verificação da influência do tipo de aleitamento na atividade elétrica dos músculos orbicular da boca, parte superior e mentual, na sucção de diferentes líquidos na fase da dentadura decídua ${ }^{(13)}$; análise das características do comportamento oral durante a sucção em crianças ${ }^{(14)}$.

Outro assunto pouco publicado é a utilização da EMG de superfície em pacientes com síndrome de Down (SD), como aparece nas pesquisas que verificaram o comportamento funcional dos músculos masseteres ${ }^{(15)}$, e análise dos músculos temporais, masseteres e supra-hióideos, direito e esquerdo, durante os movimentos de mastigação livre e com oclusão cêntrica forçada ${ }^{(16)}$. 
A criança com diagnóstico de SD apresenta alterações do sistema estomatognático, caracterizadas por diminuição de tônus e por alteração de dentição, que prejudicam as funções alimentares e a respiração ${ }^{(17-18)}$. Como estes pacientes possuem rebaixamento do tônus muscular, em diferentes graus, e desenvolvimento global mais lento(17), é importante que, além de dados da avaliação subjetiva, sejam obtidos dados de uma avaliação objetiva.

Os comportamentos miofuncionais orais esperados na sucção do neonato são: lábios vedados, compressão labial e formação de leve sulco nas comissuras labiais, movimentação dos músculos masseteres e movimentos mandibulares e ântero-posterior da língua. Qualquer mudança em uma das atividades descritas indica desvio no padrão de sucção ${ }^{(19-20)}$.

Os lábios são estruturas de grande importância na realização das funções estomatognáticas e participam da sucção, desempenhando uma importante função: a competência labial, que corresponde ao fechamento dos lábios ao redor do bico ou do mamilo, com a ação dos músculos orbicular da boca e bucinadores (responsáveis pela compressão labial e pela formação do sulco da comissura labial), de modo que o mamilo e a auréola fiquem dentro da cavidade para serem comprimidos pela língua contra o paladuro. Esse fechamento possibilita a criação da pressão intra-oral e impede que o alimento escape da cavidade ${ }^{(21-22)}$. Qualquer alteração labial, como retração, por exemplo, pode dificultar a participação dos lábios na sucção, interferindo na competência labial e na formação da pressão intra-oral ${ }^{(23-24)}$, ou ainda, resultando em escoamento do líquido ${ }^{(25)}$.

A terapia miofuncional, no trabalho com a motricidade orofacial, atua nas desordens miofuncionais, restabelecendo ou adequando as funções de respiração, de sucção, de mastigação, de deglutição e de fala. Pode-se, também, associar recursos mioterápicos, isto é, adequação da força e do movimento dos músculos a essas funções, além de remover hábitos deletérios e posturais ${ }^{(18,26-28)}$.

A hipótese que norteia esta pesquisa é delineada da seguinte maneira: crianças com SD submetidas a processo terapêutico fonoaudiológico para a adequação da função labial apresentam diferença indicativa de melhora na função muscular, por meio de análise eletromiográfica de superfície, quando comparadas com as do grupo controle. Dessa forma, esse estudo teve o objetivo de, por meio da utilização da eletromiografia de superfície, detectar a eficácia do tratamento miofuncional oral na adequação da movimentação labial, durante a sucção, em bebês com síndrome de Down.

\section{MÉTODOS}

\section{Participantes}

Nesta pesquisa, participaram 16 bebês divididos em dois grupos. O grupo pesquisa (GP) foi constituído por oito bebês com síndrome de Down (cinco do gênero masculino e três do feminino), com idade média de sete meses e 29 dias. Os critérios de inclusão deste grupo foram: descartar a hipótese de mosaicismo; ter idade inicial de seis a dez meses de vida; ter acompanhamento pediátrico; ausência de cardiopatia e não ter orientação fonoaudiológica prévia.

No grupo controle (GC), participaram oito bebês com desenvolvimento neuropsicomotor típico (cinco do gênero masculino e três do feminino), com idade média de oito meses e sete dias. Os critérios de inclusão foram: ter a mesma idade inicial do GP e sem queixa de alimentação.

\section{Material}

Para a avaliação fonoaudiológica, utilizou-se o Protocolo de Avaliação do Sistema Estomatognático do Laboratório de Investigação Fonoaudiológica em Síndromes e Alterações Sensório-Motoras (LIF-SASM) do Departamento de Fisioterapia, Fonoaudiologia e Terapia Ocupacional da Faculdade de Medicina da Universidade de São Paulo (FMUSP) (Anexo 1). As características observadas durante a avaliação foram: postura, tônus, mobilidade, responsividade e aspectos de órgãos fonoarticulatórios; dentes; hábitos orais; funções estomatognáticas de sucção, mastigação, deglutição e respiração; presença de reflexos patológicos orais; atenção da criança e relação entre mãe e bebê durante a alimentação, assim como a postura adotada por ambos. Os alimentos foram trazidos pelas mães, pois variavam de acordo com o que o bebê estava acostumado a comer em casa, assim como os utensílios que eram utilizados cotidianamente.

Para a avaliação e a reavaliação objetivas, utilizou-se o aparelho de eletromiografia de superfície, pertencente ao Centro de Especialização em Fonoaudiologia Clínica (CEFAC), modelo K6-I da marca Myotronics-Noromed, Inc. de oito canais. A freqüência de amostragem varia de 15 a $430 \mathrm{~Hz}$, sendo o ganho máximo $512 \mathrm{uV}$, não fazendo a conversão RMS do sinal. Na captação do sinal, utilizou-se minieletrodo fixo bipolar esférico de $\mathrm{Ag} / \mathrm{AgCl}$ de $2 \mathrm{~mm}$ de diâmetro, cada pólo separado por $1 \mathrm{~cm}$ de distância, como sugerido pela ISEK ${ }^{(28)}$, e eletrodo terra fixo de $\mathrm{Ag} / \mathrm{AgCl}$ de 4 $\mathrm{mm}$ de diâmetro, cujo fabricante é Alfamedic $®$. Para facilitar esta captação de sinal, utilizou-se gel condutor, também fabricado pela Alfamedic®, álcool, algodão e esparadrapo de $1,6 \mathrm{~cm}$ de largura da marca $3 \mathrm{M}^{\mathrm{TM}}$.

Na avaliação eletromiográfica dos movimentos do músculo orbicular da boca, utilizou-se papinha da marca Nestlé® etapa 1 (a partir do sexto mês), mamadeira da marca Kuka® e bico de mamadeira da marca Kuka ${ }^{\circledR}$ fase 1 (fornecidos pela Kuka Produtos Infantis Ltda.), com formato ortodôntico e furo para líquidos ralos sem aumento (oferecida a todos os bebês que participaram da pesquisa).

Para o registro de dados coletados durante o processo terapêutico, utilizou-se um protocolo de relato diário de terapia, videofilmadora marca JVC® Compact VHS e fitas de vídeo cassete VHS da marca JVC®.

\section{Procedimento}

Essa pesquisa foi aprovada pela Comissão de Ética para Análise de Projetos de Pesquisa (CAPPesq) da Diretora Clínica do Hospital das Clínicas da FMUSP sob protocolo $\mathrm{n}^{\circ}$ 753/02 e utilizado o Termo de Consentimento Pós-informa- 
ção Livre e Esclarecido referente a cada grupo participante da pesquisa: GP e GC.

Para o GP, a pesquisa foi realizada em três fases:

Na primeira fase foram realizados: a) avaliação inicial do sistema estomatognático de acordo com o protocolo de avaliação fonoaudiológica do LIF-SASM, realizada com o bebê sentado adequadamente no colo da mãe, apoiado totalmente; b) exame de EMG de superfície, que analisou o comportamento do músculo orbicular da boca em três provas: repouso habitual, retirada da papinha da colher e sucção de mamadeira. É importante salientar que nenhuma das crianças encontrava-se em fase de aleitamento materno exclusivo.

Durante o exame, o acompanhante ficava sentado em uma cadeira com encosto vertical e os pés apoiados num tapete de borracha. O bebê ficava no colo do acompanhante e a pele da região onde se colocou os eletrodos era limpa com algodão embebido em álcool. O eletrodo terra foi localizado no músculo esternocleidomastoideo, e o mini-eletrodo fixo com esparadrapo convencional no músculo orbicular da boca, na região central da porção superior. Esta porção foi escolhida pela maior facilidade em fixar o eletrodo.

Primeiramente, realizava-se a prova de repouso labial habitual, depois a retirada da papinha da colher e, por fim, a sucção de água da mamadeira. Cada prova deveria ser repetida três vezes e cada testagem tinha duração de quinze segundos. As repetições são indicadas à obtenção de uma resposta mais precisa, pois se sabe que o eletrodo de superfície mede a resposta elétrica de um grupo muscular, e não somente de um músculo específico. Durante a testagem, a pesquisadora oferecia os alimentos aos bebês, enquanto outra fonoaudióloga manuseava o aparelho. A prova do repouso labial habitual foi realizada apenas uma vez devido à dificuldade em manter os bebês numa mesma posição, sem se mexer.

A segunda fase correspondeu ao processo terapêutico, que teve duração de seis meses, com frequiência quinzenal e com sessões de quarenta e cinco minutos cada. Em média, foram realizadas doze sessões para cada criança. Nestas sessões, foram realizadas orientações e exercícios para todos os bebês, seguindo-se propostas consagradas na área ${ }^{(18,26)}$ juntamente com os cuidadores, respeitando-se o desenvolvimento individual:

1) adequação de postura do bebê: manter o bebê no colo ou em cadeirinha, o mais vertical possível, com simetria em linha média entre cabeça, cintura escapular e tronco, tanto para o uso de colher quanto de mamadeira;

2) adequação dos utensílios utilizados: tamanho da colher adequado ao tamanho da boca do bebê; a quantidade de alimento na colher deveria ser a suficiente apenas para cobrir o fundo, sem transbordar; apresentar a colher sempre de frente para a boca, em posição horizontal, de forma que o alimento tocasse levemente o lábio superior e pressionasse a ponta da língua em direção intra-oral; nova quantidade somente deveria ser ofertada após a deglutição da porção já oferecida; bico de mamadeira ortodôntico, com furo de tamanho adequado ao tipo de líquido ofertado;

3) massagens intra e extra-orais: com o dedo indicador enluvado, fazer deslizamentos horizontais em região vestibular, junto às bordas gengivais da maxila e da mandíbula, da frente para trás; fazer movimentos rotatórios na região vestibular dos músculos bucinadores; pressionar ponta da língua em direção intra-oral, com seguida oclusão de mandíbula; movimento para baixo do músculo orbicular da boca superior e para cima do músculo orbicular da boca inferior, pressionando ambos após haver feito o contato e a oclusão; deslizamento externo, com pressão, pelos músculos bucinadores, na direção orelha-comissura labial;

4) estimulação de órgãos fonoarticulatórios para trabalhar a mudança de textura alimentar, com o objetivo de evitar engasgos e de facilitar a mastigação bilateral: realizar as massagens intra-orais com o dedo enluvado embebido em líquidos e pastosos com sabores e temperaturas diferentes; posicionar, alternadamente à direita e esquerda, pequenos pedaços de legumes cozidos, bolachas macias e frutas em região posterior da gengiva inferior, com leve pressão oferecida pelo dedo enluvado e facilitar a movimentação da mandíbula para a movimentação repetida de abertura e fechamento; preparar a deglutição com facilitação para oclusão labial.

$\mathrm{Na}$ terceira fase foi realizada a avaliação final, com procedimento semelhante à inicial, após período de seis meses, aproximadamente (considerou-se um intervalo de vinte dias antes e após a data prevista).

Todas as sessões de terapia fonoaudiológica e as avaliações clínicas, inicial e final, foram filmadas, e as orientações de cada sessão foram transcritas.

Para o GC, o procedimento contou com duas fases: as avaliações inicial e final, obedecendo o mesmo procedimento realizado com o GP.

Todo procedimento foi realizado no LIF-SASM do Departamento de Fisioterapia, Fonoaudiologia e Terapia Ocupacional da FMUSP, exceto a avaliação eletromiográfica, realizada no CEFAC, para onde os pacientes foram encaminhados e acompanhados pela terapeuta.

\section{Análise estatística}

Realizou-se o teste ANOVA, que possibilitou a comparação de médias utilizando-se a variância e o Intervalo de Confiança. Definiu-se como nível de significância o valor de 0,05 e os intervalos de confiança foram construídos com 95\% de confiança estatística. Os cálculos para estabelecimento das médias foram realizados a partir dos valores em $\mathrm{uV}$ obtidos para cada sujeito em cada prova.

\section{RESULTADOS}

\section{Dados da avaliação eletromiográfica}

Compararam-se, primeiramente, os resultados das três provas (repouso habitual, mamadeira e papinha), verificando-se a diferença estatística entre as avaliações inicial e final, para o GC (Figura 1 e Tabela 1) 


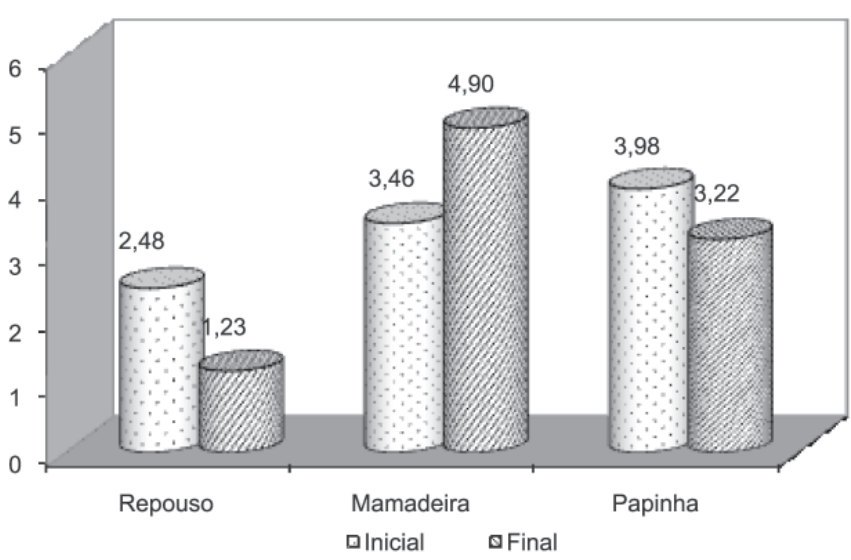

Figura 1. Comparação entre as avaliações inicial e final do GC, para as três provas

Tabela 1. Comparação das avaliações inicial e final do GC

\begin{tabular}{lcccccc}
\hline Controle & \multicolumn{2}{c}{ Repouso } & \multicolumn{2}{c}{ Mamadeira } & \multicolumn{2}{c}{ Papinha } \\
& Inicial & Final & Inicial & Final & Inicial & Final \\
\hline Média & 2,48 & 1,23 & 3,46 & 4,90 & 3,98 & 3,22 \\
Desvio padrão & 2,93 & 0,63 & 1,49 & 3,82 & 1,95 & 1,53 \\
\hline p-valor & 0,165 & 0,194 & 0,178 \\
\hline
\end{tabular}

Não foram encontradas diferenças médias estatisticamente significantes entre as avaliações inicial e final para o GC.

Realizou-se a mesma comparação entre as três provas para o GP, considerando-se as avaliações inicial e final (Figura 2 e Tabela 2).

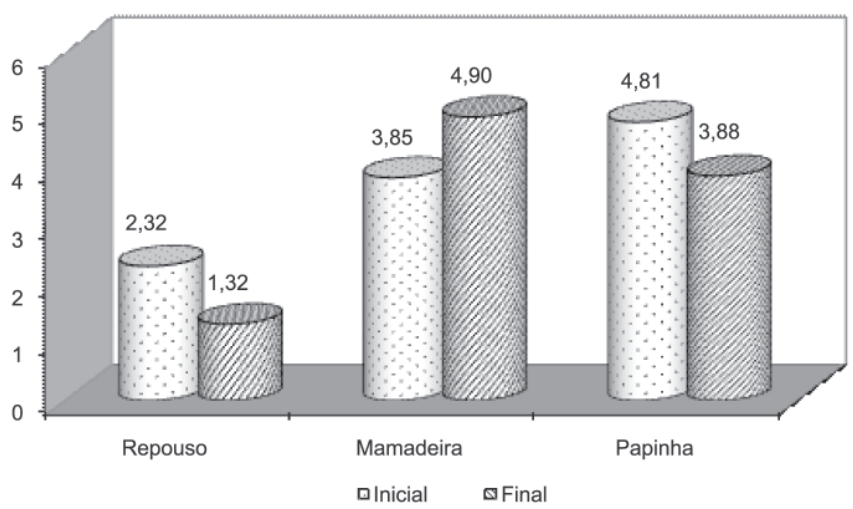

Figura 2. Comparação das avaliações inicial e final do GP, para as três provas

Tabela 2. Comparação das avaliações inicial e final do GP

\begin{tabular}{lcccccc}
\hline Pesquisa & \multicolumn{2}{c}{ Repouso } & \multicolumn{2}{c}{ Mamadeira } & \multicolumn{2}{c}{ Papinha } \\
& Inicial & Final & Inicial & Final & Inicial & Final \\
\hline Média & 2,32 & 1,32 & 3,85 & 4,90 & 4,81 & 3,88 \\
Desvio padrão & 3,08 & 0,71 & 1,74 & 3,53 & 3,59 & 2,18 \\
\hline p-valor & 0,268 & 0,300 & 0,349 \\
\hline
\end{tabular}

Assim como no GC, não foi encontrada diferença média estatisticamente significante entre as duas avaliações, em nenhuma das três provas.

Realizou-se a comparação entre grupos, considerando as respostas eletromiográficas na avaliação inicial (Figura 3 e Tabela 3).

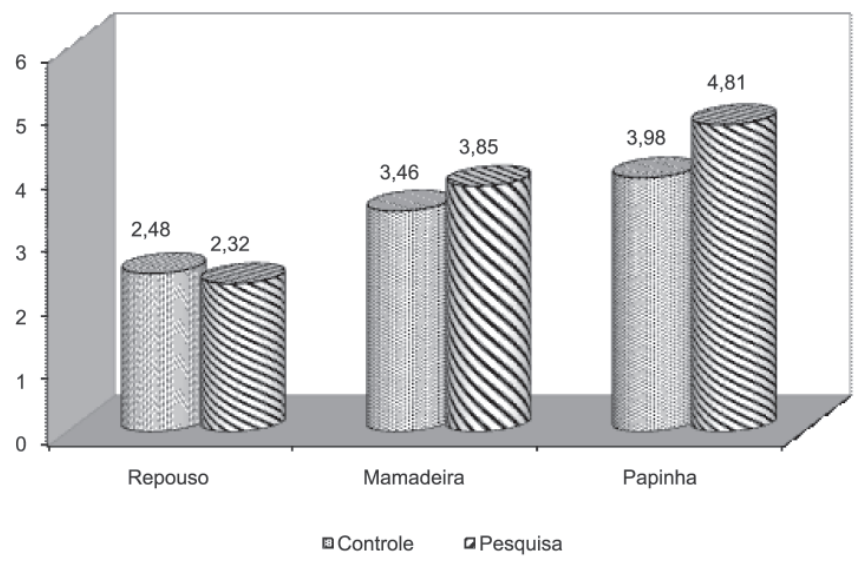

Figura 3. Comparação entre grupo, considerando as provas na avaliação inicial

Tabela 3. Comparação entre grupos, considerando as provas na avaliação inicial

\begin{tabular}{lcccccc}
\hline Inicial & \multicolumn{2}{c}{ Repouso } & \multicolumn{2}{c}{ Mamadeira } & \multicolumn{2}{c}{ Papinha } \\
& Controle & Pesquisa & Controle & Pesquisa & Controle & Pesquisa \\
\hline Média & 2,48 & 2,32 & 3,46 & 3,85 & 3,98 & 4,81 \\
Desvio & 2,93 & 3,08 & 1,49 & 1,74 & 1,95 & 3,59 \\
padrão & & & & & & \\
\hline p-valor & 0,902 & \multicolumn{2}{c}{0,524} & \multicolumn{2}{c}{0,388} \\
\hline
\end{tabular}

Não foi verificada diferença estatisticamente significante entre os grupos considerando a avaliação inicial, em nenhuma das três provas.

A mesma comparação foi realizada entre os grupos, considerando os resultados eletromiográficos da avaliação final, nas três provas (Figura 4 e Tabela 4).

Como observado, também não houve diferença estatisticamente significante entre os grupos na avaliação final, em nenhuma das três provas.

Por não haver diferença estatística entre grupos nas avaliações inicial e final, separadamente, realizou-se a mesma comparação, considerando as duas situações, simultaneamente (Figura 5 e Tabela 5).

Mesmo considerando-se as duas avaliações, simultaneamente, não foi encontrada diferença média estatisticamente significante entre os dois grupos.

A última comparação realizada foi entre as provas de repouso habitual, mamadeira e papinha, considerando-se as duas avaliações, simultaneamente. Primeiramente, realizouse a comparação no GC (Tabela 6 e Figura 6).

Para o GC existe diferença média estatisticamente significante entre as provas. Para determinar qual prova se 


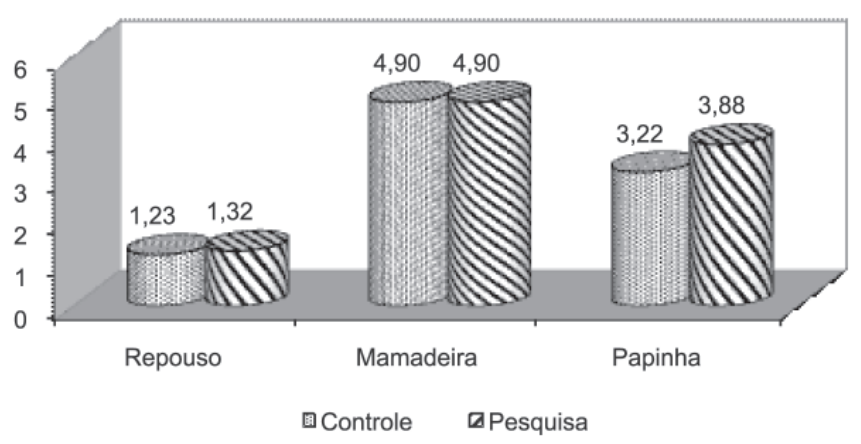

Figura 4. Comparação entre grupos, considerando as provas na avaliação final

Tabela 4. Comparação entre grupos, considerando as provas na avaliação final

\begin{tabular}{lcccccc}
\hline Final & \multicolumn{2}{c}{ Repouso } & \multicolumn{2}{c}{ Mamadeira } & \multicolumn{2}{c}{ Papinha } \\
& Controle & Pesquisa & Controle & Pesquisa & Controle & Pesquisa \\
\hline Média & 1,23 & 1,32 & 4,90 & 4,90 & 3,22 & 3,88 \\
Desvio padrão0,63 & 0,71 & 3,82 & 3,53 & 1,53 & 2,18 \\
\hline p-valor & 0,742 & \multicolumn{2}{c}{0,997} & \multicolumn{2}{c}{0,268} \\
\hline
\end{tabular}

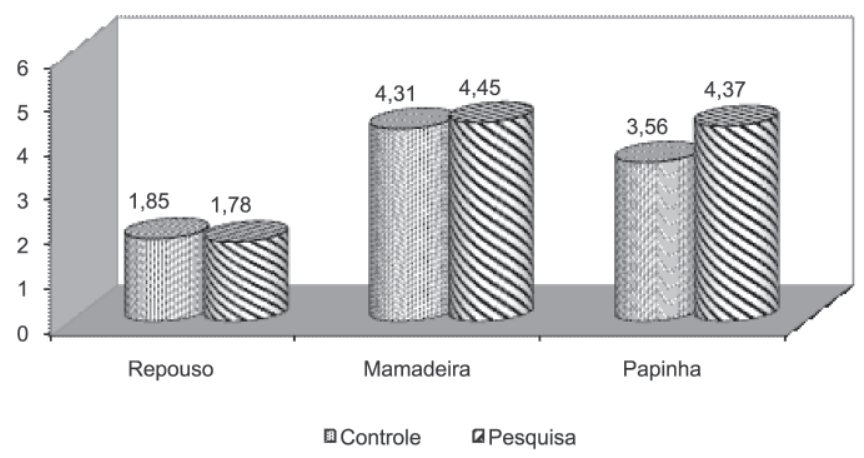

Figura 5. Comparação entre grupos, considerando as provas nas duas avaliações simultaneamente

Tabela 5. Comparação entre grupos, considerando as duas avaliações simultaneamente

\begin{tabular}{lcccccc}
\hline Geral & \multicolumn{2}{c}{ Repouso } & \multicolumn{2}{c}{ Mamadeira } & \multicolumn{2}{c}{ Papinha } \\
& Controle & Pesquisa & Controle & Pesquisa & Controle & Pesquisa \\
\hline Média & 1,85 & 1,78 & 4,31 & 4,45 & 3,56 & 4,37 \\
Desvio & 2,17 & 2,15 & 3,13 & 2,91 & 1,75 & 3,00 \\
padrão & & & & & & \\
\hline p-valor & \multicolumn{2}{c}{0,905} & \multicolumn{2}{c}{0,842} & \multicolumn{2}{c}{0,148} \\
\hline
\end{tabular}

difere, foi necessário compará-las duas a duas (Tabela 7).

A prova de repouso possui diferença estaticamente significante em relação às outras duas. Na comparação entre papinha e mamadeira, existe uma tendência à diferença, pois o p-valor está muito próximo do limite de aceitação.

A mesma comparação foi realizada com o GP (Tabela 8).

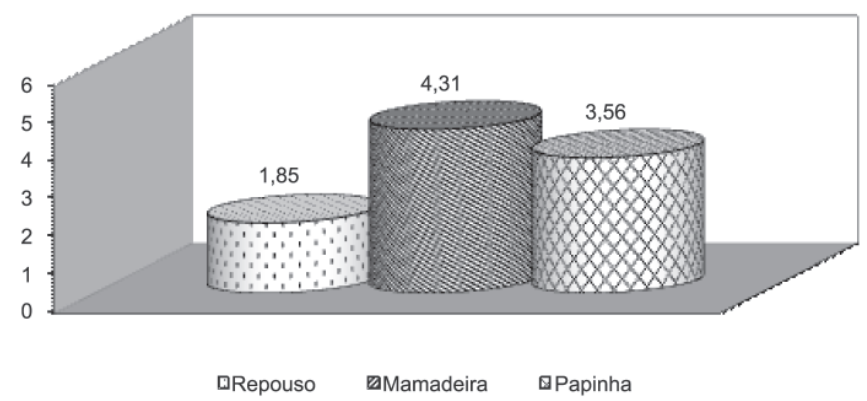

Figura 6. Comparação entre provas do GC

Tabela 6. Comparação entre provas do GP

\begin{tabular}{lccc}
\hline Pesquisa & Repouso & Mamadeira & Papinha \\
\hline Média & 1,85 & 4,31 & 3,56 \\
Desvio padrão & 2,17 & 3,13 & 1,75 \\
\hline p-valor & & 0,001 & \\
\hline
\end{tabular}

Tabela 7. Comparação duas a duas das provas do GC

\begin{tabular}{lcc}
\hline Controle & Repouso & Mamadeira \\
\hline Mamadeira & 0,002 & \\
Papinha & 0,001 & 0,203 \\
\hline
\end{tabular}

p-valores

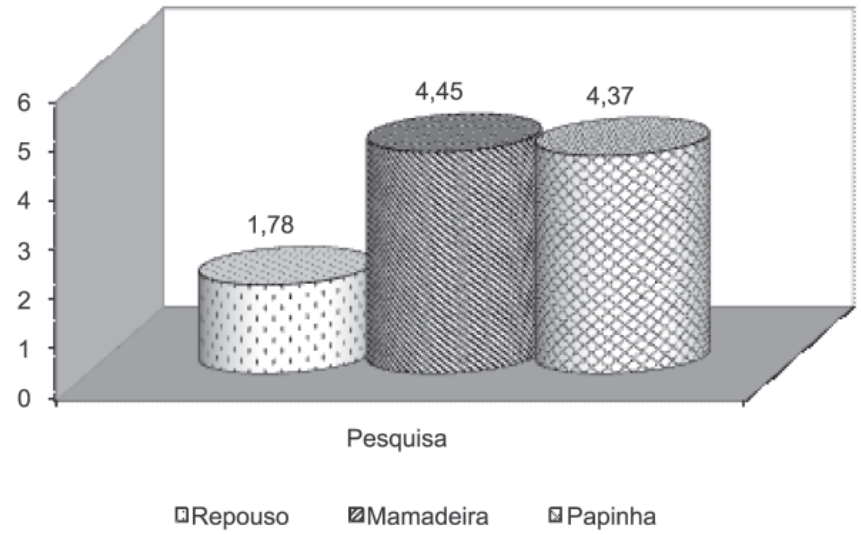

Figura 7. Comparação entre provas do GP

Tabela 8. Comparação entre provas do GP

\begin{tabular}{lccc}
\hline Pesquisa & Repouso & Mamadeira & Papinha \\
\hline Média & 1,78 & 4,45 & 4,37 \\
Desvio padrão & 2,15 & 2,91 & 3,00 \\
\hline p-valor & 0,001 \\
\hline
\end{tabular}

Da mesma forma, foi necessário comparar as provas duas a duas para o GP, para verificar qual delas se difere (Tabela 9).

Observou-se o mesmo resultado que no GC. A prova de 
Tabela 9. Comparação duas a duas das provas do GP

\begin{tabular}{lcc}
\hline Pesquisa & Repouso & Mamadeira \\
\hline Mamadeira & $<0,001$ & \\
Papinha & 0,001 & 0,908
\end{tabular}

p-valores

repouso também possui diferença estatisticamente significante quando comparada com as outras duas. Neste caso, as provas de mamadeira e papinha também apresentam tendência à diferença.

\section{Dados da avaliação clínica}

Em relação à aplicação do protocolo de avaliação do sistema estomatognático, os dados apresentados são referentes ao GP, uma vez que os bebês participantes do GC não deveriam apresentar, como não apresentaram, qualquer alteração nessa área. Os dados pertinentes para essa pesquisa foram: postura de lábios e de língua; tônus de lábios, de língua e de bochechas (Quadros 1 e 2).

\section{DISCUSSÃO}

Considerando-se a ação do músculo orbicular da boca durante a sucção, nossos dados indicam que não houve aumento significativo na atividade elatromiográfica desse mús-

Quadro 1. Postura de lábios e língua nas avaliações inicial e final do GP

\begin{tabular}{|lcccc|}
\hline & \multicolumn{2}{c}{ Lábios } & \multicolumn{2}{c|}{ Língua } \\
Crianças & $\begin{array}{c}\text { Avaliação } \\
\text { inicial }\end{array}$ & $\begin{array}{c}\text { Avaliação } \\
\text { final }\end{array}$ & $\begin{array}{c}\text { Avaliação } \\
\text { inicial }\end{array}$ & $\begin{array}{c}\text { Avaliação } \\
\text { final }\end{array}$ \\
\hline S1 & alt & alt & adeq & alt \\
S2 & adeq & adeq & alt & adeq \\
S3 & alt & alt & alt & adeq \\
S4 & adeq & adeq & adeq & adeq \\
S5 & adeq & adeq & alt & adeq \\
S6 & adeq & adeq & adeq & adeq \\
S7 & adeq & adeq & alt & adeq \\
S8 & alt & alt & alt & adeq \\
\hline
\end{tabular}

Legenda: alt = alterado; adeq = adequado culo, como alguns autores também referiram ${ }^{(13)}$. Contudo, pode-se observar que, à medida em que a sucção vai sendo substituída por outras formas de alimentação, ocorre a maturação dos movimentos dos lábios e da língua ${ }^{(21)}$.

Outra comparação realizada em nosso estudo foi entre grupos, considerando as respostas das avaliações inicial e final, separadamente. O resultado obtido foi semelhante entre os grupos, demonstrando não ter diferença na contração do músculo orbicular da boca. Em outras pesquisas observou-se o mesmo resultado, sendo que os autores sugeriram uma incoordenação muscular e não diferença no tônus, quando se refere aos músculos masseter e temporal ${ }^{(16)}$ ou uma diferença eletromiográfica que poderia ser vista em movimentos que exigissem maior potencial de ação muscular ${ }^{(15)}$. Esta, talvez, seja uma questão a ser estudada ao se considerar a movimentação do músculo orbicular da boca durante a sucção, como foi nosso objetivo.

A última comparação realizada foi entre as provas de repouso habitual, mamadeira e papinha, considerando-se as avaliações inicial e final, simultaneamente. Independente do grupo avaliado, tanto nos bebês com SD, quanto nos que apresentam desenvolvimento típico, a prova de repouso habitual teve resultados estatisticamente diferentes das outras duas, significando que a contração do músculo orbicular da boca, nessa situação, difere da condição de movimentação observada nas demais provas. Nesse sentido, alguns autores $^{(29)}$ afirmam que durante o repouso não há atividade muscular e outro estudo ${ }^{(28)}$ ressalta que o músculo, nessa situação recruta poucas unidades motoras, apresentando atividade mínima (de 0 a $5 \mathrm{mv}$ ). Por isso, a diferença da resposta eletromiográfica entre as provas já era esperada.

$\mathrm{Na}$ comparação entre as avaliações inicial e final, nas três provas, não houve diferença estatisticamente significante entre as contrações musculares do músculo orbicular da boca captados pelo eletromiógrafo de superfície, em nenhum dos grupos participantes da pesquisa. Isto significa que não houve diferença na contração do músculo estudado, no período entre as duas avaliações, mesmo no que se refere à realização do tratamento fonoaudiológico no GP.

Os achados eletromiográficos, nesta comparação, não mostraram diferença nos padrões eletromiográficos, após tratamento fonoaudiológico, o que, segundo estudo, a melhora relacionada a essa questão pode ser considerada devido ao

Quadro 2. Tônus de lábios, língua e bochechas nas avaliações inicial e final do GP

\begin{tabular}{|c|c|c|c|c|c|c|}
\hline \multirow[b]{2}{*}{ Crianças } & \multicolumn{2}{|c|}{ Lábios } & \multicolumn{2}{|c|}{ Língua } & \multicolumn{2}{|c|}{ Bochechas } \\
\hline & Avaliação inicial & Avaliação final & Avaliação inicial & Avaliação final & Avaliação inicial & Avaliação final \\
\hline $\mathrm{S} 1$ & redução & redução & adeq. & adeq. & redução & redução leve \\
\hline S2 & redução & redução leve & redução & adeq. & redução & redução \\
\hline S3 & redução & redução leve & adeq. & adeq. & redução & redução leve \\
\hline S4 & redução & redução leve & redução & adeq. & redução leve & adeq. \\
\hline S5 & redução & redução leve & adeq. & adeq. & redução & redução leve \\
\hline S6 & redução & redução leve & adeq. & adeq. & redução & redução leve \\
\hline S7 & redução & redução leve & redução & redução leve & redução & redução \\
\hline S8 & redução & redução leve & redução & redução leve & redução & redução leve \\
\hline
\end{tabular}


tipo de aparelho utilizado, ou aos movimentos avaliados ${ }^{(28)}$. Todavia, observa-se uma melhora qualitativa após a realização do tratamento, reforçando a importância da intervenção fonoaudiológica ${ }^{(18,26-28)}$.

Durante a realização da avaliação eletromiográfica, algumas dificuldades foram encontradas: movimentação do bebê e da mãe, pois o bebê ficava apoiado nela; mobilidade dos lábios devido ao balbucio ou ao choro; e manutenção da sucção pelo tempo pré-estipulado das provas, sendo que muitas vezes não foi possível realizar três vezes cada prova (como pré-estipulado), o mesmo acontecendo com a duração de alguns testes, tendo sido menores do que quinze segundos (tempo pré-estipulado) ${ }^{(11-14)}$. Considera-se, também, a questão relacionada ao local onde o eletrodo foi posicionado: região central da porção superior do lábio. Tal opção foi feita pelo fato que, por se tratar de bebês pequenos, a escolha pelo lábio inferior poderia incorrer em dificuldade na fixação devido o tamanho e proximidade entre as estruturas musculares envolvidas na região, como o músculo orbicular da boca porção inferior e mentual. Ressaltam-se, também, outros fatores importantes como a presença de baba e o extravasamento de líquido durante a sucção, comumente observados em bebês com síndrome de Down ${ }^{(17-18)}$.

Deve-se salientar a necessidade de utilização de outro eletrodo que não o indicado pelo manual do aparelho de EMG de superfície utilizado. $\mathrm{O}$ eletrodo recomendado era grande, comparando-o à superfície do músculo analisado e considerando que os sujeitos eram bebês. Para se evitar diferenças na captação dos dados, optou-se por utilizar o mesmo eletrodo fixo nas avaliações inicial e final, em todos os bebês ${ }^{(5-6,14-15)}$.

Para evitar viés de resposta, também se utilizou o mesmo formato de bico de mamadeira, ortodôntico fase 1, com mes- mo furo, para líquidos ralos, ofertados a todos os bebês, assim como a mamadeira. Todos os bicos e as mamadeiras eram da mesma marca, pois dependendo do material da mamadeira e do bico, a sucção pode ser facilitada ${ }^{(21,30)}$. O mesmo cuidado foi tomado ao se considerar a quantidade de alimentado ofertado com a colher.

\section{CONCLUSÃO}

Os dados apresentados e analisados permitem concluir que: o exame de EMG de superfície, para as provas selecionadas, não foi adequado para verificar a diferença da contração do músculo orbicular da boca, antes e após a terapia, nem a diferença entre os grupos de crianças da faixa etária estudada; possivelmente, porque os movimentos exigidos nas provas não recrutaram unidades motoras suficientes para detectar as diferenças esperadas. A avaliação fonoaudiológica, com o objetivo voltado para o sistema estomatognático, mostrou-se importante dado de avaliação clínica, tanto inicial quanto final, uma vez que permitiu observar a evolução de bebês com SD quanto à postura dos órgãos fonoarticulatórios e função de sucção. Ressalta-se a importância de se considerar a necessidade de avaliações fonoaudiológicas clínicas voltadas para essa área ainda com maior caráter qualitativo, muito embora se observem efetivos esforços no sentido de quantificar os dados qualitativos. Muito embora não se possa negar a importância em se considerar a avaliação fonoaudiológica clínica do sistema estomatognático, vale ressaltar que outros estudos sejam realizados com a eletromiografia de superfície, de forma que esse tipo de medida possa ser utilizado como instrumento objetivo de avaliação pelo profissional fonoaudiólogo.

\begin{abstract}
Purpose: To verify the efficacy of myofunctional intervention on the sucking function of infants with Down syndrome using the surface electromyography. Methods: Five male and three female infants with ages from six to ten months were paired in a study and a control groups, which were differentiated only by the diagnosis of Down syndrome. The research was carried out in three phases: a) initial assessment (clinical evaluation of the stomatognathic system and surface electromyography - tasks of habitual rest, bottle sucking and taking puree from the spoon); b) therapeutic process; c) reassessment. The control group participated only in the first and third stages. Results: The electromyographic data showed a significant difference between habitual rest and bottle/ puree tasks. In the habitual rest, fewer motor units are recruited, in comparison to the other muscle movements. Conclusion: The surface electromyography didn't allow the identification of differences between the contractions of the mouth orbicular muscle before and after the intervention, neither between the studied groups, once the movements didn't recruit enough motor units to detect the expected differences. However, the clinical evaluation did show an improvement of the assessed aspects.
\end{abstract}

Keywords: Electromyography/utilization; Down's syndrome; Infant; Sucking behavior 


\section{REFERÊNCIAS}

1. Portney LG, Roy SH. Eletromiografia e testes de velocidade de condução nervosa. In: O'Sullivan SB, Schmitz TJ. Fisioterapia: avaliação e tratamento. 4a ed. Barueri (SP): Manole; 2004. p.213-56.

2. Rainoldi A, Melchiorri G, Caruso I. A method for positioning electrodes during surface EMG recordings in lower limb muscles. J Neurosci Methods. 2004;134(1):37-43.

3. Rigler I, Podnar S. Impact of electromyographic findings on choice of treatment and outcome. Eur J Neurol. 2007;14(7):783-7.

4. Regalo SC, Vitti M, Moraes MT, Semprini M, Felício CM, Mattos Mda $\mathrm{G}$, et al. Electromyographic analysis of the orbicularis oris muscle in oralized deaf individuals. Braz Dent J. 2005;16(3):237-42.

5. Marchiori SC, Teló E, Tomé MC, Farret MM, Farret AM. An electromyographic study of the mentalis, upper and lower orbicularis oris muscles in Angle's Class II division I malocclusion. Braz J Morphol Sci. 1999;16(2):149-53.

6. Murray KA, Larson CR, Logemann JA. Electromyographic response of the labial muscles during normal liquid swallows using a spoon, a straw, and a cup. Dysphagia. 1998;13(3):160-6.

7. Yamaguchi K, Morimoto Y, Nanda RS, Ghosh J, Tanne K. Morphological differences in individuals with lip competence and incompetence based on electromyographic diagnosis. J Oral Rehabil. 2000;27(10):893-901.

8. Peláez A, Gallego G, Jiménez ID. Comportamiento de la actividad electromiográfica del músculo orbicular superior de los labios en niños con y sin labio y paladar hendido. CES Odontol. 2006;19(2):25-9.

9. Sassi FC. Eletromiografia de superfície e promoção da fluência: estudos sobre a efetividade do tratamento [tese]. São Paulo: Faculdade de Medicina da Universidade de São Paulo; 2003. 180p.

10. Sakashita R, Kamegai T, Inoue N. Masseter muscle activity in bottle feeding with the chewing type bottle teat: evidence from electromyographs. Early Hum Dev. 1996;45(1-2):83-92.

11. Tamura Y, Matsushita S, Shinoda K, Yoshida S. Development of perioral muscle activity during suckling in infants: a cross-sectional and followup study. Dev Med Child Neurol. 1998;40(5):344-8.

12. Gomes CF, Trezza EMC, Murade ECM, Padovani CR. Surface electromyography of facial muscles during natural and artificial feeding of infants. J Pediatr (Rio de J). 2006;82(2):103-9.

13. Jacinto SR. Influência do tipo de aleitamento na atividade elétrica dos músculos orbicular da boca - parte superior e mentoniano, na sucção de diferentes líquidos na fase da dentadura decídua [tese]. Piracicaba: Faculdade de Odontologia de Piracicaba da Universidade Estadual de Campinas/Piracicaba; 2000.

14. Nyqvist KH, Färnstrand C, Eeg-Olofsson KE, Ewald U. Early oral behaviour in preterm infants during breastfeeding: an electromyographic study. Acta Paediatr. 2001;90(6):658-63.

15. Niccoli Filho WD. Análise eletromiográfica do músculo masseter em indivíduos portadores da síndrome de Down [tese]. Piracicaba: Faculdade de Odontologia de Piracicaba da Universidade Estadual de Campinas/ Piracicaba; 1986.

16. Rossi Júnior WC. Análise eletromiográfica dos músculos temporal, masseter e suprahioideos em portadores da síndrome de Down [tese]. Piracicaba: Faculdade de Odontologia de Piracicaba da Universidade Estadual de Campinas/Piracicaba; 1999.

17. Mustacchi Z. Síndrome de Down. In: Mustacchi Z, Peres S, organizadores. Genética baseada em evidências: síndromes e heranças. São Paulo: CID Editora; 2000. p. 817-94.

18. Schwartzman MLC. Aspectos do desenvolvimento motor oral e da alimentação. In: Schwartzman JS, organizador. Síndrome de Down. 2a ed. São Paulo: Memnon/Mackenzie; 2003. p. 155-66.

19. Cattoni DM, Neiva FCV, Zackiewicz DV, Andrade CRF. Fonoaudiologia e aleitamento materno: algumas contribuições. Pró-Fono. 1998;10(1):45-50.

20. Neiva FCB. Proposta de um formulário de avaliação da sucção de recém-nascidos. Pró-Fono. 2000;12(2):113-9.

21. Rudolph CD. Feeding disorders in infants and children. J Pediatr. 1994;125(6 Pt 2):S116- 24. Review.

22. Lawrence R. The clinician's role in teaching proper infant feeding techniques. J Pediatr. 1995;126(6):S112-7.

23. Glass RP, Wolf LS. A global perspective on feeding assessment in the neonatal intensive care unit. Am J Occup Ther. 1994;48(6):514-26.

24. McBride MC, Danner SC. Sucking disorders in neurologically impaired infants: assessment and facilitation of breastfeeding. Clin Perinatol. 1987;14(1):109-30.

25. Morris SE, Klein MD. Pre-feeding skills: a comprehensive resource for feeding development. Tucson: Therapy Skill Builders; 1987.

26. Hennequin M. La masticación y la deglución: guía para padres y profesionales. Rev Síndrome de Down. 2000;17(2):45-7.

27. Schievano D, Rontani RM, Bérzin F. Influence of myofunctional therapy on the perioral muscles. Clinical and electromyographic evaluations. J Oral Rehabil. 1999;26(7):564-9.

28. Merletti R. Standards for reporting EMG data. J Electromyogr Kinesiol. 1999;9(1):III-IV.

29. Basmajian JV, De Luca CJ. Muscles alive: their functions revealed by electromyography. 5th ed. Baltimore: Williams \& Wilkins; c1985.

30. Hayashi Y, Hoashi E, Nara T. Ultrasonographic analysis of sucking behavior of newborn infants: the driving force of sucking pressure. Early Hum Dev. 1997;49(1):33-8.

Anexo 1. Avaliação do sistema estomatognático e postura do LIF-SASM

Nome:

Data de Nascimento:_ Idade:

Data do exame:

HD:

1) Lábios:

$\begin{array}{lll}\text { Postura no repouso: } & \text { ( ) ocluídos } & \text { ( ) ocluídos c/ contração do m. mentual } \\ & \text { ( ) entreabertos } & \text { ( ) abertos }\end{array}$

Tônus muscular:

( / ) adequado

( / ) aumentado

( / ) diminuído

Mobilidade:

( ) protrusão

( ) retração lateral D

( ) retração lateral E

( ) estalo

( ) retração lateral simétrica

( ) contração

( ) vbração

Sensibilidade:

( ) adequada

( ) reduzida

( ) aumentada 
2) Língua:

$\begin{array}{llll}\text { Postura no repouso: } & \text { ( ) papila } & \text { ( )interdentalizada } & \text { ( ) assoalho bucal } \\ \text { Tônus: } & \text { ( ) apoiada nos incisivos ( ) marca de dentes } & \\ \text { Mobilidade: } & \text { ( ) lateralização interna } & \text { ( ) lateralização externa } & \text { ( ) aumentada } \\ & \text { ( ) protrusão } & \text { ( ) estalo } & \text { ( ) elevação em ponta } \\ & \text { ( ) abaixamento } & \text { ( ) vibração } & \text { ( ) retração } \\ \text { Sensibilidade: } & \text { ( ) adequada } & \text { ( ) reduzida } & \text { ( ) aumentada } \\ \text { 3) Bochechas: } & & & \\ \text { Tônus: } & \text { ( ) adequado } & \text { ( )aumentado } & \text { ( ) diminuído } \\ \text { Mobilidade: } & \text { ( ) infla } & \text { ( ) suga } & \text { ( ) simetria } \\ \text { Sensibilidade: } & \text { ( ) adequada } & \text { ( ) reduzida } & \text { ( ) aumentada } \\ \text { Sensibilidade: } & \text { ( ) adequada } & \text { ( ) reduzida } & \text { ( ) aumentada } \\ & \text { ( ) simetria } & \text { ( ) assimetria } & \end{array}$

4) Palato mole e úvula:

$\begin{array}{llll}\text { Aspectos: } & \text { ( ) adequado } & \text { ( ) alterado } \\ \text { Úvula: } & \text { ( ) normal } & \text { ( ) desviada à } & \text { ( ) bífida } \\ \text { Palato mole: } & \text { ( ) boa mobilidade } & \text { ( ) mobilidade reduzida }\end{array}$
5) Palato Duro:
( ) adequado
( ) estreito ou ogival
( ) alto
( ) presença de fístula
6) Amígdalas:
( ) ausentes
( ) normais
( ) hipertróficas

7) Frênulos: do lábio superior:

do lábio inferior:

da língua:

8) Dentes:

Higiene e conservação dos dentes:

$\begin{array}{lll}\text { Presença de cáries: } & \text { ( ) sim } & \text { ( ) não } \\ \text { Dentição: ( ) decídua } & \text { ( ) mista } & \text { ( ) permanente } \\ \text { Ausência de dentes: } & \text { ( ) sim } & \text { ( ) não. Quais? }\end{array}$

Oclusão: ( ) adequada ( ) aberta
( ) overjet
( ) obremordida
( ) prognatismo
( ) em topo

9) Hábitos Orais:

10) Preferência Alimentar:

11) Sucção:

Vedamento labial:

Preensão do bico com canudo:

Eficiência e força:

( ) forma bico ( ) contrai bucinadores ( ) movimentação vertical de mandíbula

( ) movimentação póstero-anterior de mandíbula

( ) movimentação vertical da mandíbula

Ritmo: ( ) adequado ( ) lento ( ) acelerado

Coordenação com respiração e deglutição:

Utensílios utilizados:

Tempo de alimentação:

OBS:

Copo ( preensão, retirada do líquido): 
12) Mastigação:

Alimento utilizado:

Preensão do alimento:

Tamanho do pedaço/ quantidade introduzido na boca:

( ) lábios ocluídos ( ) lábios abertos

Movimentação de língua:

Contração de masseteres: ( ) forte ( ) fraca ( ) simétrica ( ) assimétrica

Contração de temporais: ( ) forte ( ) fraca ( ) simétrica ( ) assimétrica

Movimentação da mandíbula:

Uso preferencial: ( ) lado direito ( ) lado esquerdo ( ) não há

Ritmo: ( ) adequado ( ) lento ( ) acelerado

Coordenação com a respiração e a deglutição:

Tempo de alimentação:

Eficiência:

Colher ( tamanho, forma, material, preensão, retirada do alimento)

OBS:

13) Deglutição ( líquidos/pastoso/sólidos) :

Líquidos

Pastoso

Sólido

Coordenação com a respiração:

OBS:

14) Presença de reflexos patológicos orais:

15) Atenção da criança durante a alimentação:

16) Relação mãe e bebê durante a alimentação:

17) Respiração: ( ) nasal ( ) oral ( ) mista

18) Postura:

Da criança durante a alimentação:

Postura do cuidados durante a alimentação da criança:

Como a mãe manipula a criança:

Como o alimento é oferecido à criança:

Estratégias utilizadas pelo cuidador para um posicionamento mais funcional:

Presença de reflexoss patológicos posturais: ( ) RTL ( ) RTCA ( ) TRCS

OBS: 